\title{
Partial masking in electrocutaneous sensation: A model for sensation matching, with applications to loudness recruitment
}

\author{
W. D. LARKIN and M. J. PENNER \\ University of Maryland, College Park, Maryland
}

\begin{abstract}
A model for partial masking and other threshold-elevation effects is presented in the context of a sensation-matching paradigm. The model is applied to an electrocutaneous experiment in which the subjects adjusted stimulus intensity on the right-hand fingertip to match sensation levels of standard stimuli presented to the left fingertip. Concurrent mechanical stimulation on the right fingertip masked sensation magnitude in a way consistent with the model. Similarities between this tactile masking effect and analogous auditory phenomena are explored. When applied to loudness matching, the model describes the general shape of loudness contours and it shows that the steep slopes observed in auditory masking and "recruitment" can be a consequence of a threshold shift alone, without a supranormal growth in loudness. The model also shows that a small response bias can distort plots of sensation matching, leading to the suggestion that some varieties of loudness recruitment may not have a sensory basis.
\end{abstract}

The masking effect of one stimulus upon another is often studied by a method of sensation matching. The subject's task is to adjust the level of a partially masked stimulus so that the sensation it produces is the same as that of a stimulus not being masked. This sensation-matching paradigm is the primary focus of this paper. We present a simple model to describe the effect of partial masking on sensation magnitude in the context of the matching procedure.

Although the model may apply, in principle, to any sensory domain, it formalizes and extends an idea discussed originally in audition by Békésy (1947), Garner and Miller (1947), Licklider (1951), Steinberg and Gardner (1937). As formulated by Licklider, the hypothesis asserts that every stimulus has a "diverted" portion, that is, a portion that makes no contribution to sensory magnitude. Garner and Miller (1947) applied this notion to a problem in auditory temporal summation. Steinberg and Gardner (1937) applied it-under the rubric of a "subtractive hearing loss"- to describe the effects of noise on the loudness of a pure tone, and Békésy (1929) used it to interpret auditory fatigue. Békésy (1947) regarded the reduced sensitivity in auditory fatigue as a "subtraction" from an available pool of sensory units. We shall regard the "diverted input" more generally as a reduction in stimulus effectiveness which may be brought about by masking, fatigue, or a sensory deficit, and which is exposed

This research was supported by a grant from the National Institutes of Health (NINCDS 17170). We are grateful to Sandra Gordon-Salant, Walt Jesteadt, Scott Parker, and Robert Schlauch for helpful comments on earlier versions of this paper. We thank Rhona Hellman for providing data for Figure 8. Both authors contributed equally to this work. Correspondence may be addressed to W. D. Larkin, Department of Psychology, University of Maryland, College Park, MD 20742. by an elevation of threshold. Our purpose in this paper is not to defend any particular neurophysiological interpretation of the diverted input, but to explore a simple model based upon the idea that a loss of sensitivity at threshold is the principal factor-and perhaps the only factorresponsible for the shape of sensory-matching functions. The idea of a diverted input or a subtractive loss, in the form invoked by Békésy (1947) and by Steinberg and Gardner (1937), has been rejected by some (e.g., Harris, 1953; Stevens, 1966), but apparently not on grounds of decisive experimental evidence. One purpose of our analysis is to show that the subtractive model cannot yet be overruled.

Although partial masking phenomena have been studied mainly in vision and audition, the hypotheses we advance here can, in principle, apply to any sensory domain. To make the fundamental ideas clear, we shall apply them to an electrocutaneous sensation-matching experiment. The experiment demonstrates a tactile masking phenomenon analogous to partial masking in other sensory modalities, but which has not been a topic of previous systematic study. This experiment is described in Section 1.

In Section 2, we introduce two elaborations of the basic model. These elaborations are needed to adapt the model to any sensory domain, such as audition, in which the stimuli to be matched occupy a wide dynamic range. The first of these is a "range proportionality" assumption suggested by the work of Lim, Rabinowitz, Braida, and Durlach (1977). The second elaboration, incorporating a suggestion of Cross (1974), is designed to account for the effects of response bias in matching tasks. Together with the diverted-input hypothesis, these ideas define a general model for sensory-magnitude matching. The implications of this general model for the study of auditory partial masking, loudness recruitment, and other 
phenomena studied by means of the sensation-matching paradigm are discussed in Section 3.

\section{TACTILE MASKING: AN ELECTROCUTANEOUS DEMONSTRATION}

To begin, we present an electrocutaneous experiment in which the subject's task was to adjust a test stimulus on the right fingertip to match the sensation magnitude of a standard stimulus on the left fingertip. This contralateral matching procedure is analogous to experiments sometimes performed in audition (e.g., Hellman \& Zwislocki, 1964). The analysis of interest compares data from a condition without masking with data from conditions in which the electrocutaneous sensation was partially masked by sensations arising from purely mechanical stimulation of the skin. The masking effect of mechanical stimulation on the electrocutaneous detection threshold has been studied previously (Reilly \& Larkin, 1983; Rollman, 1974). The masking effect of mechanical stimulation on suprathreshold, nonpainful electrocutaneous sensation magnitude, which we refer to here as the tactile masking effect, has not been previously reported. Tactile masking, at threshold and above, is of interest for the information it may provide about the neural basis of transcutaneous electrical stimulation. This interest, however, is not the primary concern of this paper. For present purposes, a similar demonstration experiment could have been conceived in any sensory modality for which discrete contralateral sensations can be produced and compared. Electrocutaneous stimuli are not unique in this regard but, because there is no history of previous data, or of theoretical argument about what results are to be expected, the experiment provides a neutral context in which to demonstrate sensory-matching phenomena and the diverted-input model.

The stimuli used in the electrocutaneous experiment were brief capacitive discharges applied to the fingertip. These stimuli generate action potentials in afferent sensory neurons by depolarizing the nerve membrane at or near its nerve endings. Psychophysical responses to these stimuli therefore closely mirror the excitation parameters for single cutaneous neurons (Larkin \& Reilly, 1984; Pfeiffer, 1968; Rollman, 1975). Because these stimulating conditions are radically different from the normal excitation of receptors in vision, audition, and other senses, the experiment serves to demonstrate that certain general properties of sensation-matching data can be understood without reference to properties of specific mechanical or photochemical transducers.

\section{Method}

Stimuli and Apparatus. The stimuli were discharges from a 200-pF capacitor, applied to the fingertip (2nd digit). A single discharge was presented each time the subject touched a spherical electrode (3.4- $\mathrm{cm}$ diameter). Each contact with the electrode drained its stored charge through a ground path provided by the subject's body and a return electrode strapped to the ankle. The stimulus current and voltage waveforms show a rapid rise at the beginning of electrical contact followed by an exponential decay that drops to $1 / \mathrm{e}$ in less than $5 \mu \mathrm{sec}$. The evoked sensations are described as ranging from "light touch" to "pinprick," "jab," and "pain," depending on the amount of charge transferred from the electrode to the skin. (These sensations resemble those from common electrostatic discharges, such as the "carpet sparks" encountered in dry, upholstered environments. In the case of carpet sparks, however, an electrical charge stored on the surface of the human body is discharged to a metal object, such as a doorknob, rather than the other way round.) The apparatus has been documented fully by Reilly and Larkin (1983). Readers interested in techniques for measuring and controlling the stimuli are directed to that paper, and to Larkin and Reilly (1984) for details that would consume inordinate space if repeated here.

Procedure. In the basic paradigm, the subject adjusted the stimulus level for the right-hand, middle fingertip so that it matched the sensation magnitude felt when a fixed, standard stimulus was applied to the left fingertip. These adjustments were made by means of a smoothly turning, unmarked potentiometer knob. The potentiometer was linked in series with an experimenter's control which could be reset randomly before each trial to ensure that the subject's knob would not have a predictable range and rate of change. The subject was instructed to bracket the matching stimulus before making a final setting.

On each trial, one of eight standard stimulus levels was randomly selected for presentation to the left fingertip. The lowest of these was close to absolute threshold, but was always detectable. The highest was just below the subject's pain threshold. The subject first sampled the standard stimulus by touching the electrode three to five times with the left fingertip, and then said "ready." Within $1 \mathrm{sec}$ the experimenter reset the stimulus to a randomly chosen low level, and the subject immediately began to touch the electrode with the right fingertip, while adjusting the stimulus to a value that matched the standard. The rate of stimulation was self-paced, but regular in tempo, with a maximum of about 20 stimuli per minute. Each trial ended when the subject announced a satisfactory match. Typically, a match was announced after 30 to $90 \mathrm{sec}$ of adjustments. This protocol differs from many auditory procedures in that the standard stimulus was not constantly interpolated between successive presentations of the test stimulus. It was almost always sufficient to sample the standard stimulus only at the beginning of each trial. On the rare occasions when this procedure was not sufficient, the subject requested the trial to be restarted.

The left-to-right matches were carried out in at least six 1.5-h sessions, after one or two practice sessions. In each session, the subject made matches in a calibration condition and in at least one of three masking conditions, which differed according to the amount of contact force used when stimulating the right fingertip.

In both the calibration and the masking conditions, the stimuli were delivered by tapping the electrode with a practiced, stereotypical motion of the fingertip. Each tap produced a momentary period of mechanical contact (dwell time: $80-100 \mathrm{msec}$ ) and a controlled force of contact. The contact force was measured by means of an accelerometer mounted inside the electrode housing, and was displayed to the subject as a decibel reading on an analogue meter dial. (See Reilly \& Larkin, 1983, for details of the force-monitoring apparatus.) For the calibration condition, taps on the electrode were executed with a constant, minimum controllable force, which registered as " $0 \mathrm{~dB}$ " on the dial. This condition minimized the sensations that arise from simply touching the electrode, and rendered the masking effects of touch unmeasurable (Reilly \& Larkin, 1983). For the masking conditions, the same minimum contact force was maintained on the left fingertip (standard stimulus), but taps with the right fingertip were executed with greater force, corresponding to $\pm 20, \pm 40$, or $\pm 50 \mathrm{~dB}$ on the accelerometer. These more forceful taps, which masked the sensations from the electrical discharge, will be referred to as "light," "medium," and "strong" 
taps, respectively. The $\pm 50-\mathrm{dB}$ tap is the strongest that can be maintained with consistency throughout an entire experimental session. The subjects were required to execute the taps within a 2-dB margin of error at each level of tap force. The subject was instructed to equate the sensation magnitude of the left and right electrocutaneous stimuli, without regard for the sensations arising from mechanical contact.

\section{Results and Discussion}

We present data for two adult male participants, designated S1 and S2. Each had participated in other electrocutaneous experiments and was highly practiced in the adjustment task.

Calibration data. The left-to-right calibration matches were well described by simple power functions, fitted by least squares to the log-transformed data in each separate session $\left(\mathrm{S} 1, r^{2}>\right.$.98; $\left.\mathrm{S} 2, r^{2}>.99\right)$. There were no significant differences among the session $\times$ session exponents, so the calibration data were combined across sessions, separately for each subject. Power functions fitted to the combined data gave an exponent of .88 for $S 1\left(r^{2}\right.$ $=.998)$ and .96 for $S 2\left(r^{2}=.996\right)$. The data and fitted power function for $\mathrm{S} 1$ are shown in Figure 1.

Neither the excellent fit of the power function nor the departure of the exponent from unity (in the case of S1) was unexpected: both findings are typical of sensory matching in the absence of a masking stimulus (Marks, 1974; Stevens, 1975), and both have been reported previously in a similar electrocutaneous matching task (Larkin \& Reilly, 1986). The session-to-session stability of the calibration matches is an important result, because it permits these data to be used as a baseline for a study of the effects of masking.

Before turning to the masking results, we note that asymmetry in calibration matches (as measured by an exponent different from unity) could, in principle, stem from either a left/right sensory disparity, a response bias, or both. For $\mathbf{S 1}$, whose exponent was .88, supplementary data support the response-bias interpretation because (1) detection thresholds were not different for the two hands, and (2) reversing the roles of standard and comparison stimuli, by presenting them to the opposite hands,

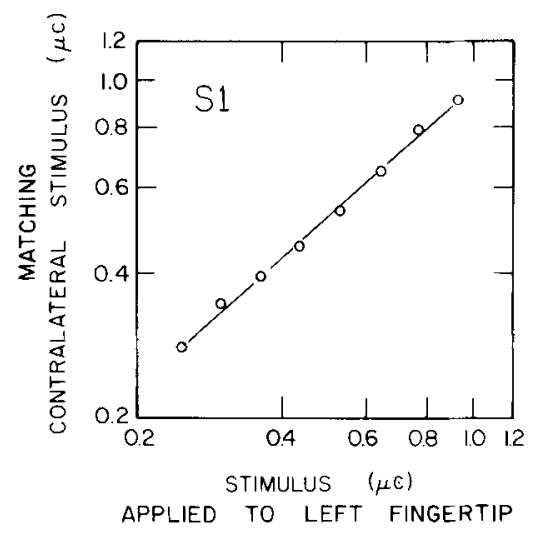

Figure 1. Results of electrocutaneous calibration matching, without tactile masking, for Subject S1. The data are averaged for six sessions. The fitted power function has an exponent of .88 .

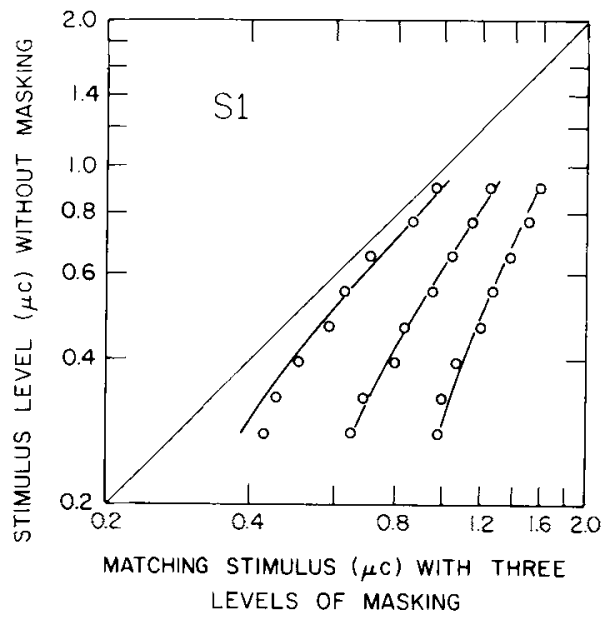

Figure 2. Results of electrocutaneous sensation matching with three levels of tactile masking for Subject S1. Each point represents a pair of stimuli judged to produce the same sensation magnitude. Stimuli plotted horizontally were partially masked by concurrent mechanical stimulation. Stimuli plotted vertically were not masked. From left to right, three masking conditions are shown, corresponding to "light," "medium," and "strong" taps on the electrode. Theoretical curves represent least-squares fitting of Equation 1.

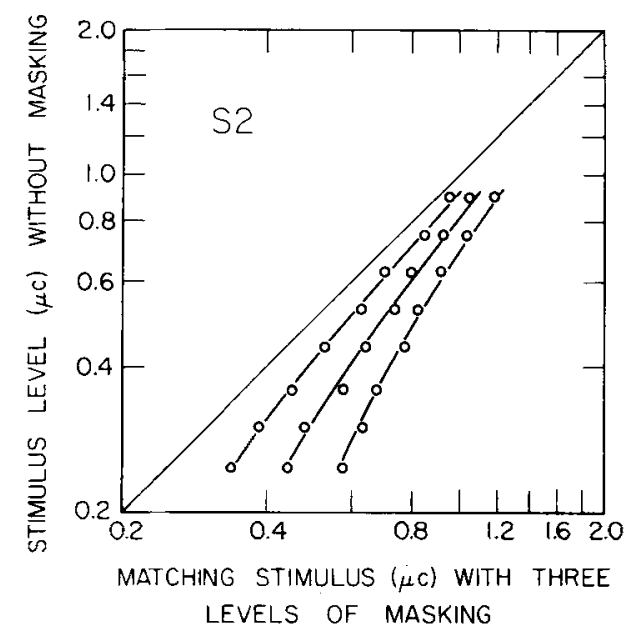

Figure 3. Results of electrocutaneous sensation matching with three levels of tactile masking for Subject $\mathbf{5 2}$. The data are plotted as in Figure 2.

gave an exponent close to the reciprocal of .88 when plotted as in Figure 1. In what follows, we shall assume that any response bias present in the calibration conditions is also present in the tactile masking conditions. Two different methods of dealing with response bias are described in this paper. One, which we adopt here, is simply to use the calibration data as a reference against which the responses under tactile masking are measured. A second method is to employ a model that permits separate estimates of response bias and of masking effects. We shall present this model in Section 2.

Masking data. Figures 2 and 3 show results for the three masking conditions. The horizontal axis plots the matching stimulus (amount of electrical charge, in 
microcoulombs) needed on the right fingertip for sensations masked by three levels of contact force. The vertical axis shows the stimulus needed to produce the same sensations without masking (i.e., the electrical charge on the right fingertip at the 0 - $\mathrm{dB}$ tap force, as determined by calibration results like those depicted in Figure 1). If there were no tactile masking effect, all the data in Figures 2 and 3 would lie precisely along the diagonal. That they do not so lie cannot be attributed to response asymmetry, because only right-fingertip sensitivity is plotted.

Figures 2 and 3 give the visual impression that tactile masking increases the rate of growth of electrocutaneous sensation, as there is an increase in steepness for each increase in masking level. This interpretation is analogous to the "recruitment" interpretation of loudness-balance plots in audition. Loudness recruitment, an apparently supranormal growth of loudness, is said to occur for unilateral hearing loss whenever there is a diminishing difference in loudness between a normal and an abnormal ear as the stimulus level increases. The steepness of a loudness-balance plot, analogous to Figures 2 and 3, or the equivalent laddergram representation of the slope, is taken as the standard measure of recruitment (Brunt, 1985; Carver, 1978; Hallpike, 1976; Sanders, 1979). ${ }^{1} \mathrm{Be}-$ cause of the wide dynamic range usually available in audition, a logarithmic (decibel) scaling of the stimuli is advantageous in plotting loudness data. For electrocutaneous stimuli, however, there is no compelling technical advantage in a logarithmic plot, so we have replaced the logarithmic coordinates of Figures 2 and 3 with linear coordinates in Figure 4. Here, the data follow roughly parallel lines fitted separately by least squares to each masking condition. None of the slopes differs significantly from 1.0. If there is any departure from colinearity, it is both visually and statistically unimpressive, with $r^{2}>.98$ in all cases. ${ }^{2}$ Tactile masking produces a shift away from the $45^{\circ}$ diagonal, but the plots remain essentially parallel: there is no diminishing difference between sensation magnitudes for masked and nonmasked stimuli as the level of stimulation rises.
The purpose of comparing Figures 2 and 3 with Figure 4 is to demonstrate that, without a logarithmic transformation of the units of measurement, the steepness of sensation-matching functions may not show any dependence on the degree of masking. Of course, the physical range of electrocutaneous stimulation is inherently narrow. The smaller the dynamic range, the more likely that a unit slope will typify the data when graphed as in Figure 4. Nevertheless, it is clear that, whether the tactile masking effect is displayed on linear or logarithmic coordinates, it can be well represented as an additive translation of the sensation-matching function. That is, if the stimulus levels required for a given sensation magnitude are denoted by $X_{m}$ with masking and by $X_{o}$ without, then the sensation-matching plots are described by

$$
X_{m}=X_{o}+K,
$$

where $K$ is a constant representing the degree of masking, and both $X$ and $K$ are measured in microcoulombs.

To judge the fit of Equation 1, a least squares estimate of $K$ was computed for each masking condition, and the resulting lines of unit slope were superimposed on the data, as shown in Figures 2 and 3. The estimates of $K$ were larger for $S 1$ than for $S 2$, but the statistical fits were nearly equivalent. The average residual sum-of-squares (SSR) in fitting Equation 1, expressed as a proportion of the total sum-of-squares (SS), was .022 for S1 and .011 for S2. Because of the rather narrow dynamic range for electrocutaneous stimulation, the curvature of Equation 1 is not a pronounced feature of these logarithmic plots, which might alternatively suggest a set of power functions. However, power functions do not radically change the statistical adequacy of fit: averaged SSR/SS values were $45 \%$ smaller for each subject when power functions (each with two free parameters) were substituted for Equation 1. This reduction in residual variance does not represent a statistically significant improvement in fit, whether it is regarded as applying to each masking condition separately $[F(1,6)=5, p>.05]$ or to each set of three masking experiments $[F(3,21)=5.8, p>.05 / 3]$.

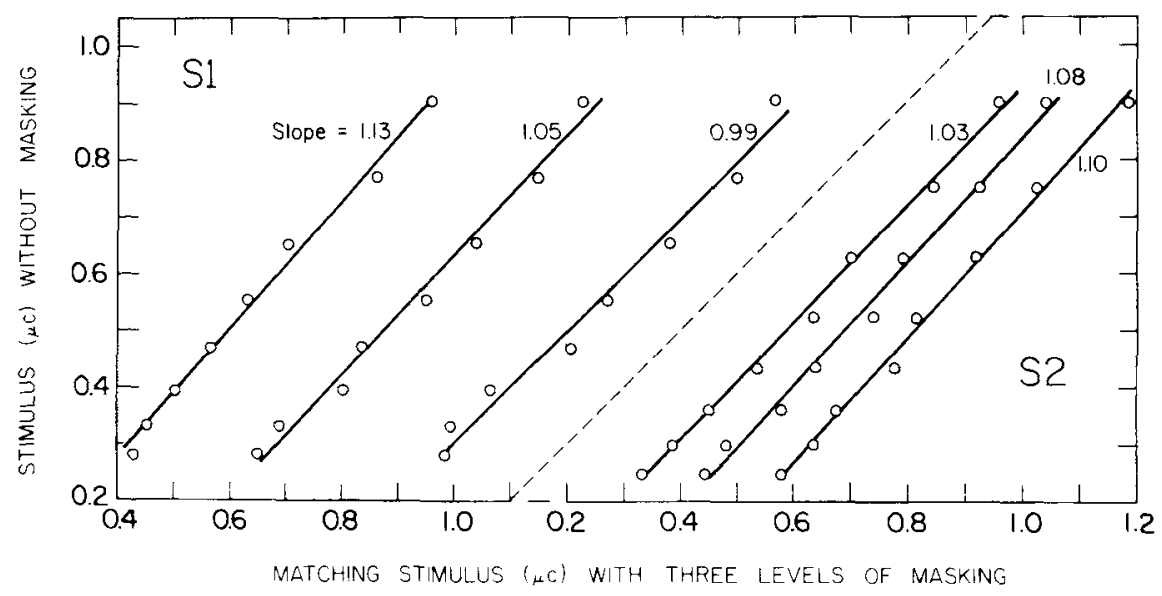

Figure 4. Data from Figures 2 and 3, replotted on linear coordinates. Straight lines have been fitted by least-squares, without constraining their slopes or intercepts. 
The parallelism displayed in Figure 4 would be expected if the physical range of stimulation, from threshold to pain tolerance, were invariant under different levels of tactile masking. A constant dynamic range would be consistent with the common observation that vigorous tactile stimulation can elevate pain thresholds. This analgesic effect has been documented for a variety of stimulating conditions (Higgins, Tursky, \& Schwartz, 1971; Wall \& Cronly-Dillon, 1960). Pertovaara (1979) has shown that the effect depends upon the specific activation of Pacinian afferents. If subtolerance levels of stimulation are muted uniformly, as Figure 4 shows, then it is at least plausible that the upper limit of sensation could also submit to the same adjustment, leaving an unaltered dynamic range.

It is important to notice that this range-invariance hypothesis will not appear to be confirmed if data consistent with it are viewed on a logarithmic plot: the range translation implied by Equation 1 is not preserved under a logarithmic transformation. To make this assertion clear, consider a pair of stimuli, $X_{a}$ and $X_{b}$, defining a range, $R=X_{b}-X_{a}>0$. The translation defined by Equation 1 shifts the boundaries of the range to new values, $X_{a}^{\prime}=$ $X_{a}+K$, and $X_{b}^{\prime}=X_{b}+K$, so that the new range, obviously, is unchanged in linear units:

$$
R^{\prime}=X_{b}^{\prime}-X_{a}^{\prime}=R .
$$

However, in logarithmic units, the range is not invariant:

$$
\left(\log X_{b}^{\prime}-\log X_{a}^{\prime}\right)<\left(\log X_{b}-\log X_{a}\right) .
$$

Consequently, if Equation 1 describes the effects of masking, then the masked stimulus will appear to have a compressed range when the data are viewed on logarithmic coordinates, as demonstrated in Figures 2 and 3.

The foregoing elementary observation about the logarithmic transformation shows that the apparent steepness of a sensory-matching function does not in itself provide an unambiguous measure of the rates of growth of sensation: any conclusion about rates of growth must be tied to a deliberate choice of a measurement scale. For our transcutaneous electrical stimuli, there are compelling reasons to believe that the total charge transferred from the electrode to the skin, measured in microcoulombs (rather than, e.g., the stimulus energy or the current amplitude), is the appropriate measure (Larkin \& Reilly, 1984; Reilly, Freeman, \& Larkin 1985). A logarithmic transformation of stimulus charge has no fundamental (measurement-theoretic) justification and, unlike the decibel scale in audition, it usually is not needed as a matter of technical convenience. For these reasons, we conclude that the suprathreshold tactile masking effect simply shifts the dynamic range of stimulation uniformly, in accord with Equation 1. For the stimuli we have studied, masking does not effect a more rapid growth of sensation.

This conclusion applies specifically to tactile masking from mechanical stimulation, and not necessarily to other manipulations that may also reduce electrocutaneous sensitivity. For example, cooling the skin surface also raises the detection threshold for these stimuli, but the suprathreshold effect is decidedly multiplicative: sensitivity is attenuated to a much greater degree at levels far above threshold than at levels close to it (Larkin \& Reilly, 1986).

The masking effectiveness of mechanical stimulation apparently depends upon the kinetic energy of the tap. At the electrocutaneous threshold, the degree of masking is nearly independent of the size and shape of the object touched, and therefore is not intimately related to mechanical pressure on the skin (Reilly \& Larkin, 1983). Our past experiments, together with those of Rollman (1974), show that tactile masking at threshold does not depend on whether the mechanical stimulus is delivered passively or is controlled by the subject. In the suprathreshold case studied here, the subject's role in controlling tap force leads to the question of whether the masking effect is purely a sensory phenomenon or might be influenced by the motor program (or the "effort function") for executing the tap. This ambiguity cannot be resolved without doing further experiments of a different kind. The theory advanced here, of course, is that the degree of masking is uniform from threshold to the highest levels of stimulation. The good fit of the theory suggests to us that any sensorimotor contribution may be negligible.

Because electrocutaneous sensitivity is governed by the charge contained in any brief, monophasic stimulus, and not by details of the stimulus waveform, the masking effects observed here should generalize to any comparably brief pulses. Much remains to be learned about the physical requirements for an effective mechanical masker, but because this question goes beyond our present concern, we turn next to a general model for matching, and then to its application in the domain of loudness.

\section{THE BASIC MODEL AND ITS ELABORATIONS}

The linear-shift pattern shown in Figure 4 and described by Equation 1 can be related to the diverted-input hypothesis (Licklider, 1951). This hypothesis holds that for any level of stimulation, $X$, there is some portion, $\tilde{X}$, that is ineffective, in that it does not contribute to the resulting sensation. Thus, the effective portion of a stimulus is $X$ $-\tilde{X}$. If two stimuli, $X_{1}$ and $X_{2}$, are matched in sensation magnitude when their "effective levels" are the same, then the matching requirement.

$$
X_{1}-\tilde{X}_{1}=X_{2}-\tilde{X}_{2},
$$

is equivalent to

$$
X_{2}=X_{1}+K,
$$

which is just a restatement of Equation 1, with $K=$ $\tilde{X}_{2}-\tilde{X}_{1}$.

Equation 5 represents the diverted-input hypothesis in its most basic form. Note that Equation 5 cannot apply if $X_{1}$ and $X_{2}$ have different dynamic ranges. A more general condition for stimulus matching is that the effec- 
tive levels of the stimuli occupy the same proportion of their available ranges. Denoting these ranges by $R_{1}$ and $R_{2}$, respectively, a match occurs when

$$
\frac{X_{1}-\tilde{X}_{1}}{R_{1}}=\frac{X_{2}-\tilde{X}_{2}}{R_{2}} .
$$

Let $\varrho=R_{2} / R_{1}$, and redefine $K$ as $K=\left(X_{2}-\varrho X_{1}\right)$. Then the sensory matching function is given by

$$
X_{2}=\varrho X_{1}+K \text {. }
$$

The case in which $\varrho=1$ is of special interest, because it represents the linear-shift pattern seen in the electrocutaneous data. Figure 5 displays examples of this case, plotted on logarithmic coordinates over a wide dynamic range. This plot exhibits various choices of the threshold differential, $K$, ranging from 10 to $60 \mathrm{~dB}$. The lower asymptote of each curve indicates the degree of threshold elevation in $X_{2}$, relative to $X_{1}$. Despite the strong curvature in the logarithmic plot, the rate of change of $X_{2}$ is actually constant and precisely matched to that of $X_{1}$; that is, $\mathrm{d} X_{2} / \mathrm{d} X_{1}=\varrho=1$.

When $\varrho \neq 1$, Equation 7 provides that the relative rates of growth of sensory magnitudes for $X_{1}$ and $X_{2}$ are determined by their respective ranges: sensation grows less for a unit increase in the stimulus with the larger range than for a unit increase in the stimulus with the smaller range. This property of the model is in qualitative agreement with theory and data given by Teghtsoonian (1971), in his analysis of psychophysical functions. ${ }^{3}$ The notion that the shape of the matching function depends upon the ranges of the stimuli being matched was used by Lim et al. (1977) as a way of normalizing the integration of jnds. Our matching condition, Equation 6, is a simplified adaptation of their range-normalization hypothesis.

The idea that psychophysical judgments can depend upon the boundaries of the stimulus range has, of course, a long history in psychology. In Parducci's (1974) work, for example, the range effect is assumed to depend upon the stimuli an experimenter elects to present, rather than

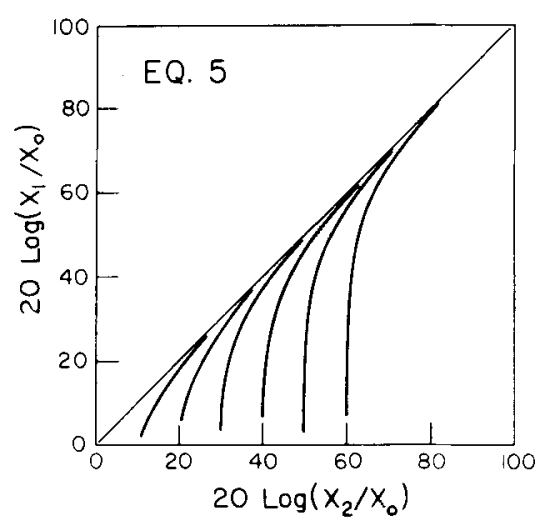

Figure 5. Model of sensation matching, plotted in decibels, for the case of equal stimulus ranges. Curves are shown for six values of the threshold-shift parameter, $K$, in Equation 5. The arbitrary reference level, $X_{0}$, corresponds to $0 \mathrm{~dB}$.

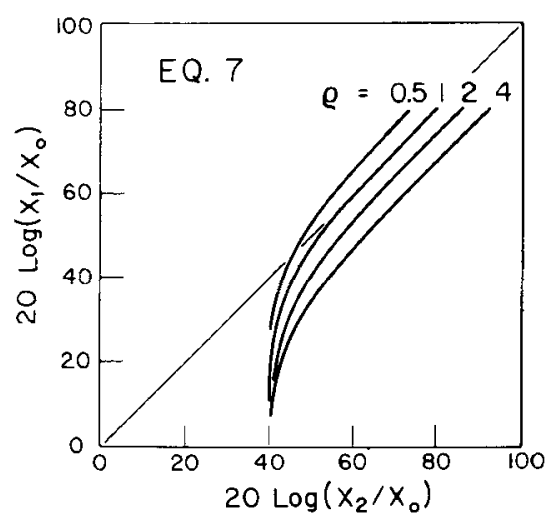

Figure 6. The model for sensation matching (Equation 7), plotted in decibels for various degrees of range inequality. $A$ 40-dB threshold elevation in $X_{2}$, relative to $X_{1}$, was assumed in each case.

upon the dynamic limits of the sensory apparatus. Whether e should be interpreted as an experiential or as a neurosensory parameter is a question we leave for future investigation (see Penner \& Larkin, 1989).

When $\varrho \neq 1$, the logarithmic plots of Equation 7 approach a laterally shifted diagonal at high levels, but they retain a fixed lower asymptote. A set of examples is shown in Figure 6, for a 40-dB threshold elevation in $X_{2}$, relative to $X_{1}$. The lateral shift amounts to $6 \mathrm{~dB}$ for each doubling (or halving) of $\varrho$, resulting in curves that either cross the main diagonal $(\varrho<1)$, or fail to reach it $(\varrho>1)$. Over a stimulus range as large as that in Figure 6, a small inequality of range can be virtually impossible to detect in experimental data. For example, if the range of $X_{2}$ is only $90 \%$ of the range of $X_{1}$, (i.e., $e=.9$ ), the difference between the shifted and the unshifted curves at high levels amounts to only $1 \mathrm{~dB}$. This insensitivity to small differences in range means that we can apply the simpler, one-parameter model in many situations, taking $\mathrm{e} \approx 1$ as a robust approximation. In Section 3, however, we shall demonstrate one application of the more general model.

The matching hypothesis represented by Equation 7 can be applied directly to the electrocutaneous data (Figures 2 and 3 ) because, in these experiments, the subject's response bias was cancelled by referring all responses to a calibration condition. If this is not done (i.e., if one simply plots the stimulus level on the right against the matching stimulus level on the left), the possibility of some response asymmetry (as in Figure 1) must also be taken into account. Response asymmetry of this kind is prevalent in psychophysical matching procedures (Cross, 1974; Marks, 1974; Stevens, 1975). For example, Houtsma, Durlach, and Braida (1980) found that 2 subjects with normal hearing produced differences as large as $20 \mathrm{~dB}$ in interaural loudness matching, depending on whether the subjects adjusted the stimulus to the left or the right ear. To illustrate how such behavioral effects might be taken into account, consider the ubiquitous "regression effect" found in a variety of psychophysical judgment tasks (Hol- 
lingworth, 1910; Stevens \& Greenbaum, 1966). Subjects tend to compress their responses to a stimulus under their control, relative to the range of a standard stimulus controlled by the experimenter. Cross (1974) has shown that this tendency can be described by allowing a powerfunction exponent to take alternate values: one value when the left stimulus is adjusted to match the right, and a different value when the right stimulus is adjusted to match the left. In the present context, regression bias can be represented by the following generalization of Equation 7:

$$
X_{2}=\alpha\left(\varrho X_{1}+K\right)^{\beta} \text {, }
$$

where $\alpha>0$ and $\beta>0$ represent the effects of response bias. In Equation 8, any departure of $\beta$ from unity signifies a compression of the range of responses given to one stimulus, relative to the other. The parameter $\alpha$ is a necessary scaling factor, which depends upon the value of $\beta .^{4}$

The form of Equation 8 is familiar enough in psychophysics to require a note of caution: the exponent, $\beta$, is introduced here as a response parameter, not as a measure of sensory transduction. We expect $\beta$ to be independent of $K$ and, perhaps, to reflect some aspects of the experimental protocol (as does the response criterion in signal-detectability theory), as well as the inherent behavioral tendencies of the individual. One way to estimate $\beta$ would be to arrange an experimental situation in which $K$ can be set to zero. If the model is correct, such an estimate could then be applied to data for the same individual in any similar matching task with a nonzero value of $K$.

If the stimulus range is narrow, as it is in any electrocutaneous experiment, only an extreme value of $\beta$ can effect a radical difference between Equations 7 and 8 . For stimuli with wide dynamic ranges, the difference can be substantial, even if $e$ is close to 1.0. This comparison is depicted in Figure 7. Equation 8 is plotted here on logarithmic coordinates over a $100-\mathrm{dB}$ range; the solid

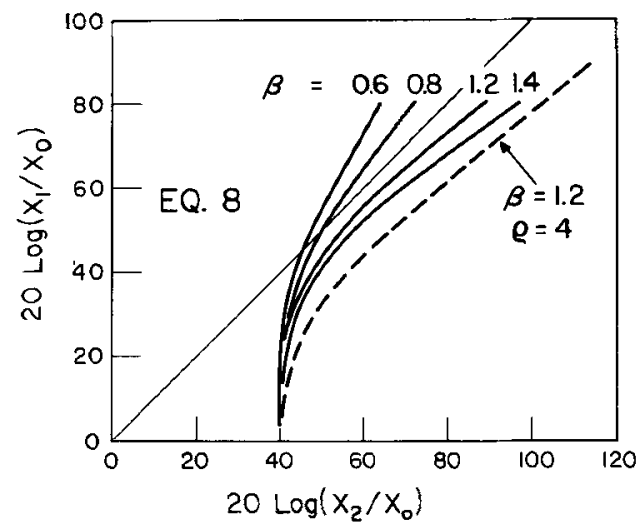

Figure 7. The model for sensation matching (Equation 8), plotted in decibels for varions degrees of response bias. A 40-dB threshold elevation was assumed for $X_{2}$, relative to $X_{1}$. Solid lines indicate the equal range condition: $e=1$. Dashed line shows one example for biased responding with unequal ranges. lines indicate $\varrho=1.0$. As in Figure 6, a 40-dB threshold shift has been assumed. Despite the relatively small departures of $\beta$ from unity, the curve is shifted by several decibels and no longer converges to the diagonal. The dashed line illustrates one case of unequal stimulus ranges $(\varrho=4)$, combined with a biasing exponent $(\beta=1.2)$. Note that the effect of the range inequality is to shift the curve uniformly at high levels, just as in Figure 6.

It is clear from Figures 6 and 7 that the sensationmatching function can take a variety of shapes, depending upon the degree of inequality of the stimulus ranges and the degree of asymmetry in the subject's response habits. The larger the dynamic range of a matching experiment, the more these effects are exposed. Therefore, we next consider what the general model, or its special cases, would imply about loudness matching in audition, in which the dynamic range is typically very large.

\section{IMPLICATIONS FOR LOUDNESS MATCHING AND LOUDNESS RECRUITMENT}

In this section, we shall consider three areas of auditory measurement in which loudness matching has a central role: the partial masking of tones by noise, the measurement of loudness recruitment in audiology, and the determination of equal-loudness contours by means of heterofrequency comparisons among pure tones. The purpose of this section is to make clear the implications of the model just presented, with attention to its empirical deficiencies, and to suggest how the model might be tested. Existing psychoacoustic and audiological data do not permit a satisfactory test, for two reasons. First, much published data are given only as averages for groups of subjects, rather than for individuals. The steep curvature shown in Figures 5, 6, and 7 can be attenuated, or concealed, by combining data for individuals who have slightly different values of the threshold parameter, $K$. While individual data are the norm in the audiological literature, the exigencies of clinical testing usually do not permit the degree of measurement precision, nor the sheer quantity of data, needed to make detailed comparisons with a model. Second, although it may be possible to fit Equation 7 to published data by regarding $\varrho, K, \alpha$, and $\beta$ as free parameters, an experimental test requires that at least some of these parameters be estimated independently of the sensory-matching procedure. For example, because $K$ is a threshold parameter, it could be estimated in a threshold procedure. Few existing studies provide this information for individual subjects-none with the precision that is required. Nor are there any studies in which the response-bias characteristics of individuals have been related to their performance in loudness matching. In a thorough test of the model, an attempt would be made to estimate its parameters in separate experimental procedures, and then to examine the degree of parameter invariance across procedures. Experiments designed to per- 
mit these comparisons are underway, and will be reported in a forthcoming paper. For the present, our assessment of the model will be largely qualitative.

\section{Partial Masking}

Examples of partial masking data selected from two experiments are shown in Figure 8. In both experiments, listeners equated the loudness of a $1000-\mathrm{Hz}$ tone in quiet with that of a $1000-\mathrm{Hz}$ tone in noise. The data in both cases are for groups of normal-hearing individuals who experienced approximately the same degree of threshold elevation when the noise was present. In the experiment by Hellman, Scharf, Teghtsoonian, and Teghtsoonian (1987), a 1.2-sec tone was presented monaurally every $2.4 \mathrm{sec}$, and noise was gated on for $2.1 \mathrm{sec}$ during alternate presentations of the tone. Wideband noise was used in one condition, narrowband noise in another. In Thalmann's (1965) experiment, only wideband noise was used: it was continuously present in one ear. The tone, $250 \mathrm{msec}$ in duration, was presented once per second, alternately to the masked and to the contralateral ear. The procedural differences between these experiments apparently were inconsequential, as the data nearly overlap in the wideband-noise condition.

We have superimposed Equation 7, with $\varrho=1$, on the data for the narrowband-noise condition in Figure 8. The least squares fit is excellent, with $r^{2}>.99$. This good fit shows that the partial masking effect in the narrowband condition can be accounted for entirely by the effect of the noise on threshold: this group of subjects equated the loudness of the tone in noise to the loudness

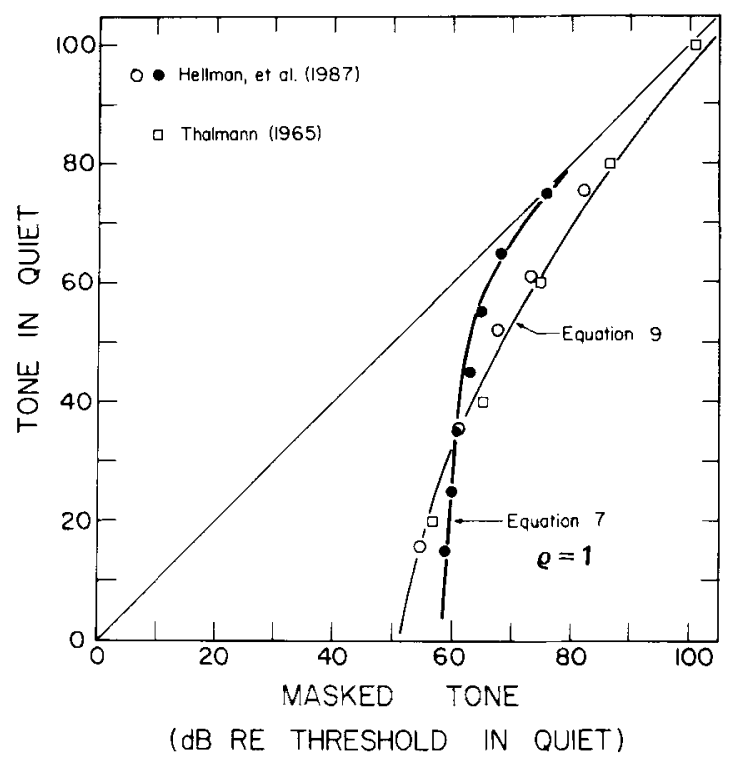

Figure 8. Data from two experiments on loudness matching. The vertical axis depicts the sensation level of a 1-kHz tone in quiet. The horizontal axis depicts the corresponding sensation level required for a judgment of equal loudness when the tone is partially masked by noise, either narrowband (solid symbols) or wideband (open symbols). On both coordinates, sensation level is indicated in $\mathrm{dB}$ relative to the threshold in quiet. (For details of plotting, see Note 5.) of the tone in quiet when both tones exceeded their thresholds by the same amount, in units of sound pressure. For reasons just outlined, we do not regard this result as a test of Equation 7, but it demonstrates that the simplest version of the diverted-input hypothesis can be descriptively accurate.

Neither Thalmann's (1965) nor Hellman et al.'s (1987) data, nor other group data we have seen for wideband masking appear to be compatible with Equation 7. Most studies (e.g., Hellman, 1970; Hellman \& Zwislocki, 1964; Lochner \& Burger, 1961; Pavel \& Iverson, 1981; Stevens \& Guirao, 1967) show group partial masking functions to be less steep than Equation 7 requires. In the wideband condition, the following generalization of Equation 7 often provides a better description of the data:

$$
X_{2}=\left(X_{1}^{a}+K^{a}\right)^{1 / a} .
$$

Taking $a \approx .3$ gives an acceptable fit to Thalmann's results, as depicted in Figure 8. Equation 9 is a special case of the partial masking functions developed by Iverson and Pavel (1981). Unfortunately, it is not yet understood why the parameter $a$ must take a value different from 1.0 in the wideband-noise case. This question, as well as the question of fitting individual data, remains for further study. Our preliminary conclusion is that Equation 7 may be valid as a limiting case in auditory partial masking experiments.

\section{Loudness Recruitment}

The plots displayed in Figures 5, 6, and 7 bear a firstorder resemblance to plots of interaural loudness balance (matching loudness in one ear to loudness in the other) when there is a unilateral impairment of the hearing mechanism (Brunt, 1985; Carver, 1978; Fritze, 1980; Miskolczy-Fodor, 1960). The steepness of the loudnessbalance plot is commonly taken as a measure of loudness recruitment in the impaired ear. The classical paradigm for the measurement of recruitment is the Alternate Binaural Loudness Balance test, introduced by Fowler (1936). In this test, the audiological patient adjusts a tone in one ear to match the loudness of a standard tone presented alternately to the other ear. Unilateral versions of the procedure, originating with Reger's (1936) seminal paper, also are in common use.

For the recruitment interpretation, $X_{1}$ represents sound pressure in the normal ear, $X_{2}$ represents sound pressure in the impaired ear, and Equation 5 represents a uniform shift in sound pressure, without any implied change in the rate of growth of loudness or in the dynamic range of auditory sensitivity. In Equation 7, as well, the rates of growth of loudness need not change, though the rate for $X_{2}$ may differ from the rate for $X_{1}$. The strong curvature shown when these equations are plotted, as in Figures 5 and 6, does not signify that the loudness of $X_{2}$ undergoes an initially rapid growth, relative to $X_{1}$, and then, at high levels, resumes a "normal" rate of growth, although this would be the classical interpretation (Brunt, 1985; Carver, 1978). Rather, the curvature is entirely a consequence of plotting sound pressure on logarithmic 
coordinates. To the extent that these plots capture the typical form of loudness-recruitment data, they show that such data signify a threshold shift, but not a supranormal growth of loudness. Working with clinical data, Sanders (1979) reached the related conclusion that there may be nothing "abnormal" about loudness recruitment.

When $\varrho \approx 1$, Equation 7 describes "complete recruitment" (Brunt, 1985) in the sense that the upper portion of the loudness-matching function appears to follow the main diagonal on a decibel plot. Clinical data that fall short of the diagonal ("underrecruitment") or that cross it ("overrecruitment") can be accommodated in these respects by taking $\varrho \neq 1$, but, as noted in Section 2 , an empirically significant degree of under- or overshoot (e.g., more than 2 or $3 \mathrm{~dB}$ ) can be achieved only if a rather large discrepancy is assumed between the ranges of $X_{1}$ and $X_{2}$. The required discrepancy is greater than that usually induced by a threshold shift alone. This means that, in the context of the present model, clinical "underrecruitment" and "overrecruitment" phenomena imply either that something much more drastic has happened to the overall range of sensitivity in the affected ear, or that the explanation lies elsewhere, in the domain of response bias.

Figure 7 makes clear that a small degree of response bias, represented as a power transformation in our model, can produce loudness-balance patterns similar to underrecruitment and overrecruitment. To test this hypothesis would require not only a quantitatively successful fit of Equation 7 to individual data, but also an independent experimental determination of the bias parameter. If a patient has a frequency-selective hearing loss, with a normal hearing threshold in some frequency range, $\beta$ could be estimated by a loudness-balance procedure carried out in the range of normal hearing. To our knowledge, such a procedure has not been used in audiological research, although there is an extensive literature on the question of asymmetries in loudness-balance testing.

There is a great variety of types and degrees of hearing loss among audiological patients, and it would be unreasonable to expect Equation 7 to suffice for every clinical category. For example, we do not expect any version of our model to apply to unilateral conductive loss (a constant attenuation factor for one ear). In the absence of response bias, conductive loss can be represented by introducing a new multiplicative parameter:

$$
X_{2}=c\left(\varrho X_{1}+K\right),
$$

where $c$ is the attenuation factor. For cases of pure conductive loss, we may take $\varrho=1$ and $K=0$. Equation 10 then would describe a family of straight lines parallel to the diagonal in a logarithmic plot. For nonzero values of $K$, Equation 10 is functionally equivalent to Equation 7 . An empirical distinction between these two cases is possible only if the parameters can be determined separately, in independent experiments. This example demonstrates, again, that the shape of a loudness-balance curve may not be a secure guide for an audiological diagnosis. The present scheme of equations provides a framework for analysis that could enhance the diagnostic value of the loudness-balance procedure.

\section{Equal-Loudness Contours}

The applications we have considered thus far have assumed that the primary sensory phenomenon exhibited by loudness-matching curves is the threshold elevation in a masked or an impaired ear. Although the threshold elevation may also induce a compression of dynamic range, requiring $\varrho \neq 1$, loudness-matching data are inherently insensitive to such effects. For example, if $X_{1}$ and $X_{2}$ are assumed to have the same upper limit, at $120 \mathrm{~dB}$ soundpressure level, but thresholds of 0 and $60 \mathrm{~dB}$, respectively, then the range of $X_{2}$ is, indeed, narrower than that of $X_{1}$, but only .1\% narrower in units of sound pressure. In a loudness-matching experiment, it is not likely that this situation could be distinguished from one in which the two ranges were identical.

To exhibit the effects of substantial differences in ranges of $X_{1}$ and $X_{2}$, we turn to heterofrequency loudness matching, that is, experiments in which the two stimuli are sinusoids of different frequencies, $f_{1}$ and $f_{2}$. For example, we may take $f_{1}=1000 \mathrm{~Hz}$ and $f_{2}=8000 \mathrm{~Hz}$. For the "standard" normal-hearing listener, the threshold for hearing a $1000-\mathrm{Hz}$ tone is about $6 \mathrm{~dB}$ below that for the $8000-\mathrm{Hz}$ tone. At the high end of loudness, a 120-db tone at $1000 \mathrm{~Hz}$ matches an $8000-\mathrm{Hz}$ tone at about $127.5 \mathrm{~dB}$ (ISO, 1961). There is very little difference between the decibel ranges of $f_{1}$ and $f_{2}$ in this example, but $f_{2}$ has the larger range by a factor of 2.37 in linear units of sound pressure. Thus, to apply Equation 7 to this example, we use 2.37 as an estimate of $\varrho$.

A range parameter, $\varrho$, was established by the method illustrated above for several $f_{1}, f_{2}$ pairs, with $f_{1}=1000 \mathrm{~Hz}$ as the constant stimulus, and the 120-dB level of $f_{1}$ as the defined upper limit of the stimulus range. To estimate $K$, the minimum audible field intensities (ISO, 1961) were taken as standard thresholds. Using these estimates, a loudness-matching function was computed for each tone pair. The results of these computations are displayed in Figure 9, in the form of predicted equal-loudness contours.

Figure 9 demonstrates that much of the familiar geometry of equal-loudness contours can be recovered from Equation 7 (i.e., from a model that accounts for loudness matching as a linear equalization of stimuli within their respective ranges). In this application of the model, estimates of $\varrho$ have a dominant role, but the method of estimating them is not critical: the results are very similar when, for example, the upper limit is taken as 110 , rather than $120 \mathrm{~dB}$.

In Figure 9, the mean (absolute) deviation of the predicted values from published equal-loudness standards (e.g., see Scharf, 1978, p. 198) is less than $5 \mathrm{~dB}$ for the frequency range $50 \mathrm{~Hz}$ to $8 \mathrm{kHz}$, and it is negligible from 8 to $12 \mathrm{kHz}$. Free-field loudness contours were used to illustrate the fit, because their undulation across the fre- 


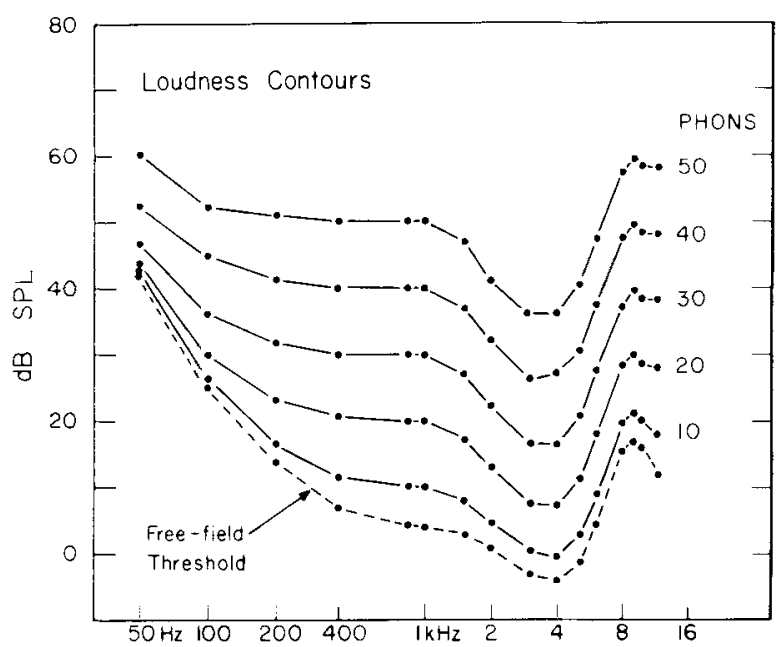

Figure 9. Predicted form of equal-loudness contours, using Equation 7. Points at $1 \mathrm{kHz}$ were used as reference standards for all other frequencies. The free-field threshold contour (broken line), and the 120 phon free-field contour (not shown) were used to estimate $\varrho$ at each frequency.

quency spectrum challenges the model rather more than the relatively flat contours for the headphone situation. The match-to-standard data is comparable to that achieved by $\mathrm{Lim}$ et al. (1977), who also used the rangeproportionality idea, but imbedded it in an hypothesis about intensity resolution. Comparison of Figure 9 with the data of Lim et al. shows that the main features of equal-loudness contours can be captured without integrating an intensity-resolution function separately at each frequency. The basic shape of the contours is determined by differences in the overall range of stimulation at different frequencies. Below $50 \mathrm{~Hz}$, the standard contours ascend more rapidly than Equation 7 allows. We presume that the same problem would be found for the Lim et al. model. This discrepancy may be related to the special problems of measurement at low frequencies (Robinson \& Dadson, 1956).

\section{DISCUSSION AND CONCLUSIONS}

The experiment reported in Section 1 demonstrates that the basic model (Equation 5) accurately describes partial masking in at least one sensory domain. Concurrent mechanical stimulation on the fingertip raises the requirement for electrocutaneous stimulation uniformly such that sensation magnitudes remain constant for stimuli that are elevated the same amount above threshold. In this case, the threshold elevation alone, rather than any nonlinear suprathreshold effect, is sufficient to account for the data. We postulate that a similar phenomenon may underlie some partial masking effects in audition, as well as the loudness-recruitment result typical of sensorineural hearing loss. We have exhibited one set of loudness-matching data, for the case of a pure tone masked by narrowband noise, that displays an excellent fit to the basic model.
We have also seen that the shape of equal-loudness contours is well predicted when stimulus ranges are taken into account.

Although we do not expect all sensory-matching phenomena to be subsumed by the equations given here, the general analysis shows that only one parameter-the threshold shift, $K$-is needed to account for the usual shape of sensory-matching functions when they are plotted on logarithmic coordinates. In addition, bias parameters, $\alpha$ and $\beta$, are needed to represent the response asymmetry that subjects may impose on their matching judgments. For any stimulus domain with a wide dynamic range, only a slight response bias can radically affect both the degree of curvature and the displacement of a sensory-matching function. These biasing effects are reminiscent of some of the special recruitment functions studied in audiology. The comparison suggests that, in some instances, recruitment may not have a sensory origin apart from a threshold difference for the two stimuli.

\section{Comparison With Other Models}

The precise form that a partial masking or a loudnessbalance plot should take has been a matter of considerable study. Stevens (1966) suggested that both could be described by power functions, the exponents of which increase with the degree of masking or threshold elevation. In this hypothesis, a loudness-matching function should consist of two straight segments in logarithmic coordinates, one segment rising from the horizontal axis toward the diagonal, and the other continuing upward along, or adjacent to, the diagonal (see Stevens \& Guirao, 1967, for examples). Stevens's hypothesis was adopted as a descriptive model in some audiological textbooks (e.g., Davis \& Silverman, 1978; Hallpike, 1976), although no compelling theoretical reasons for it have been advanced. The problem of distinguishing a pair of connected straight-line segments from various possible curves makes an empirical test of Stevens's hypothesis especially difficult. As we have seen, the degree of curvature displayed by our equations depends upon the range of stimulation employed. For stimuli with a narrow range, as in Figures 2 and 3 , the plots can resemble power functions.

Although the present model is incompatible with Stevens's (1966) hypothesis for the shape of loudnessbalance plots, it is not necessarily inconsistent with his psychophysical power law. If both sides of Equation 5 (or Equation 7, etc.) were raised to the $p$ th power, our formulation would incorporate Stevens's (1955) postulate for the growth of loudness. However, the loudness exponent, $p$, is unnecessary in the present context, because it cancels when taking logarithms on both sides of the equation (for a logarithmic plot), or when both sides are raised to the $1 / p$ (for a linear plot). The existence of a psychophysical power law is not at issue in the present formulation because the graph of loudness balance does not bear the imprint of the presumed psychophysical function. Treisman (1964) gives a cogent illustration of this fact. 
Two other theoretical approaches to partial masking and loudness-balance functions have been mentioned in earlier sections. Both studies began with assumptions very different from the diverted-input hypothesis that we have used here. Nevertheless, the form achieved in these other models is in some cases similar to the plots of our equations. Lim et al. (1977) began with a "proportional jnd" hypothesis about how the growth of loudness depended on intensity resolution, and then considered auxiliary assumptions that could produce a wide variety of curve shapes. For auditory partial masking, Iverson and Pavel (1981) invoked a mathematical requirement that families of functions must be parallel, in a generalized sense. This "shift-invariance" requirement was supported in data from monaural loudness-matching experiments in which a tone in noise was adjusted to match a tone in silence (Pavel \& Iverson, 1981). In conjunction with other assumptions, shift invariance leads to two general mathematical expressions, one of which was proposed earlier by Lochner and Burger (1961). To fit either of Iverson and Pavel's formulas to data requires estimating four parameters. In Pavel \& Iverson's (1981; Table 3) study, these estimates varied markedly from one experiment to another.

Our approach differs from that of both Lim et al. (1977) and Iverson and Pavel (1981) in two main respects. First, we propose that response factors independent of the sensory phenomena may have a nonnegligible effect. Second, we have pursued a manifestly simple hypothesis for sensation matching, not in the expectation that it would suffice for all cases of interest, but with the goal of providing a formulation more easily accessible to direct empirical tests. A separate paper (Penner \& Larkin, 1989) will report some of these tests.

\section{Units of Measurement}

The fundamental idea we have advanced is that two stimuli should be matched in sensory magnitude when they are both the same distance from their respective thresholds. An important question is, "In what units is the 'distance' to be measured?" For the electrocutaneous stimuli, we alluded to theoretical and empirical results in support of the notion that electrical charge, measured in microcoulombs, is the natural stimulus metric. For the auditory applications, we assumed that the stimulus should be specified in units of sound pressure (e.g., in Newtons $/ \mathrm{m}^{2}$ ). This aspect of our presentation may be troubling to some, who may prefer to think of pressure ratios as the natural way to measure an auditory stimulus. But the choice of a stimulus metric is not an arbitrary matter: different choices can determine one's interpretation of data and can change a simple interpretation into a complex one, or vice versa. Our use of sound-pressure differences in a model for loudness matching offers a comprehensive and simple account of a variety of auditory phenomena. Conceivably, an alternative account, based upon pressure ratios, could be developed that would also provide an integrated description of partial masking, loudness recruitment, and equal-loudness contours. In that event, by comparing the two schemes of explanation, one could make a more informed decision about the appropriateness of one stimulus metric versus the other.

Lacking such an alternative, it may be useful to remark that pressure differences have had an important role in at least two different areas of audition outside the present context. One example is Laming's (1986) study of the sensory basis for a generalized version of Weber's law. Laming found that the sensory mechanism responds to sound pressure differences, $\Delta X$, not ratios, when the intensity of one stimulus is compared with that of another. A second example is the modified sone scale for loudness (Scharf, 1978):

$$
L=k\left(X-X_{0}\right)^{0.6},
$$

where loudness, $L$, is determined by the pressure difference between a stimulus, $X$, and a reference threshold, $X_{o}$. Note that the sone scale can be correct only if the fundamental assumption of this paper is correct; that is, that two stimuli are matched in sensory magnitude when they are the same distance from their respective thresholds. A subtractive translation of the stimulus has been the topic of much discussion in the context of the psychophysical power function (Marks \& Stevens, 1968). To the extent that an expression such as Equation 11 is valid in psychophysics, one could argue that the appropriate way to measure the stimulus is in linear units of $X$, rather than as a dimensionless ratio of the form $X / X_{0}$.

\section{A Supranormal Growth of Loudness?}

In presenting this model, we offer a theoretical perspective with implications for several problems in psychophysics and audiology. For example, the widely held assumption that steeply sloping recruitment functions signify an abnormal growth of loudness has motivated a search for other evidence to corroborate this audiological diagnosis. The search has not met with much success. Both the small-increment sensitivity test and the acoustic reflex test have been suggested as giving indirect evidence of supranormal loudness growth, but neither shows a consistent relationship to recruitment data (Fastl \& Schorn, 1981; Hirsh, Palva, \& Goodman, 1954; Martin \& Brunette, 1980; Sanders, 1979; Scharf, 1976). As another example, the belief in supranormal loudness growth may lead one to expect that auditory neurons would exhibit a steeper rate response function for a tone presented against a background of noise than for a tone presented in quiet. Neurophysiological evidence, so far, does not confirm this expectation (Costalupes, Young, \& Gibson, 1984; Phillips, 1987; Salvi, Henderson, Hamerink, \& Ahroon, 1983). These and other problems would have a different status if it could be shown that steeply sloping sensation-matching functions on logarithmic coordinates 
are not an apodictic demonstration of supranormal growth. The empirical plausibility of this view is established by the data and the model we have presented.

\section{REFERENCES}

BÉKÉsY, G. voN (1929). Zur theorie des Hörens: Über die Bestimmung des einem reinen Tonempfinden entsprechenden Erregungsgebietes der Basilarmembran vermittlest Ermüdungserscheinungen. Physikalische Zeitschrift, 30, 115-125.

BÉKÉSY, G. voN (1947). The recruitment phenomenon and difference limen in hearing and vibration sense. Laryngoscope, 57, 765-777.

Birnbaum, M. H. (1973). The devil rides again: Correlation as an index of fit. Psychological Bulletin, 79, 239-242.

Brunt, M. A. (1985). Békésy audiometry and loudness balance testing. In J. Katz (Ed.), Handbook of clinical audiology (3rd ed., pp. 273291). Baltimore: Williams \& Wilkins.

CARVER, W. F. (1978). Loudness balance procedures. In J. Katz (Ed.), Handbook of clinical audiology (2nd ed., pp. 164-178). Baltimore: Williams \& Wilkins.

Costalupes, J. A., Young, E. A., \&ibson, D. J. (1984). Effects of continuous noise backgrounds on rate response of auditory nerve fibers in cat. Journal of Neurophysiology, 51, 1326-1344.

Cross, D. V. (1974). Some technical notes on psychophysical scaling. In H. R. Moskowit, B. Scharf, \& J. C. Stevens (Eds.), Sensation and measurement (pp. 23-36). Dordrecht, Holland: D. Reidel.

DAVIS, H., SilvermaN, S. R. (1978). Hearing and deafness (4th ed.). New York: Holt, Rinehart and Winston.

FASTL, H., \&CHORN, K. (1981). Discrimination of level differences by hearing-impaired individuals. Audiology, 20, 488-502.

FowLER, E. P. (1936). A method for early detection of otosclerosis. Archives of Otolaryngology, 24, 731-741.

FrITzE, W. (1980). The graph of loudness recruitment (ABLB test). Archives of Oto-Rhino-Laryngology, 226, 11-13.

Garner, W. R., Miller, G. A. (1947). The masked threshold of pure tones as a function of duration. Joumal of Experimental Psychology, 37, 293-303.

HALLPIKE, C. S. (1976). Sensori-neural deafness and derangements of the loudness function: Their nature and clinical investigation. In W. D. Keidel \& W. D. Neff (Eds.), Handbook of sensory physiology: Vol. 5, Part 3. Auditory system: Clinical and special topics (pp. 1-35). New York: Springer-Verlag.

HARRIS, J. D. (1953). A brief critical review of loudness recruitment. Psychological Bulletin, 50, 190-203.

Hellman, R. P. (1970). Effect of noise bandwidth on the loudness of a $1000-\mathrm{Hz}$ tone. Journal of the Acoustical Society of America, 48, 500-504.

Hellman, R., Scharf, B., Teghtsoonian, M., \& Teghtsoonian, $R$. (1987). On the relation between the growth of loudness and the discrimination of intensity for pure tones. Journal of the Acoustical Society of America, 82, 448-453.

Hellman, R. P., \&WISLOCKI, J. (1964). Loudness function of a 1000cps tone in the presence of a masking noise. Journal of the Acoustical Society of America, 36, 1618-1627.

Higgins, J. D., Tursky, B., \& Schwartz, G. E. (1971). Shock-elicited pain and its reduction by concurrent tactile stimulation. Science, 172, 866-867.

Hirsh, I. J., Palva, T., \& Goodman, A. (1954). Difference limen and recruitment. Archives of Otolaryngology, 60, 525-540.

HoLLINGWORTH, H. L. (1910). The central tendency of judgment. Journal of Philosophy, Psychology, \& Scientific Methods, 7, 461-469.

Houtsma, A. J. M., Durlach, N. I., \& Braida, L. D. (1980). Intensity perception: XI. Experimental results on the relation of intensity resolution to loudness matching. Journal of the Acoustical Society of America, 68, 807-813.

ISO (1961). Normal equal-loudness contours for pure tones and normal threshold of hearing under free field listening conditions (Recommendation R-226). New York: International Organization for Standardization.

Iverson, G. J., \& Pavel, M. (1981). On the functional form of partial masking functions in psychoacoustics. Journal of Mathematical Psychology, 24, 1-20.

Laming, D. (1986). Sensory analysis. London: Academic Press.

LARKIN, W. D., \& Rilly, J. P. (1984). Strength/duration relationships for electrocutaneous sensitivity: Stimulation by capacitive discharges. Perception \& Psychophysics, 36, 68-78.

LARKIN, W. D., \& REILlY, J. P. (1986). Electrocutaneous sensitivity: Effects of skin temperature. Somatosensory Research, 3, 261-271. LICKLIDER, J. C. R. (1951). Basic correlates of the auditory stimulus. In S. S. Stevens (Ed.), Handbook of experimental psychology (pp. 9851039). New York: Wiley.

Lim, J. S., Rabinowitz, W. M., Braida, L. D., \& Durlach, N. I. (1977). Intensity percpetion: XIII. Loudness comparisons between different types of stimuli. Joumal of the Acoustical Society of America, 62, 1256-1267.

LOChNer, J. P. A., \& Burger, J. F. (1962). Form of the loudness function in the presence of masking noise. Journal of the Acoustical Society of America, 33, 1705-1707.

LUCE, R. D. (1978). Dimensionally invariant numerical laws correspond to meaningful qualitative relations. Philosophy of Science, 45, 1-16.

MARKs, L. E. (1974). Sensory processes: The new psychophysics. New York: Academic Press.

Marks, L. E., Stevens, J. C. (1968). The form of the psychophysical function near threshold. Perception \& Psychophysics, 4, 315-318.

Martin, F. N., B BunetTe, G. W. (1980). Loudness and the acoustic reflex. Ear \& Hearing, 1, 106-108.

Miskolczy-Fodor, F. (1960). Relation between loudness and duration of tonal pulses: III. Responses in case of abnormal loudness function. Journal of the Acoustical Society of America, 32, 486-492.

PARducc1, A. (1974). Contextual effects: A range-frequency analysis. In E. C. Carterette \& M. P. Friedman (Eds.), Handbook of perception Vol. 2. pp. 127-141. New York: Academic Press.

Parker, S., Casey, J., Ziriax, J. M., \& SilberberG, A. (1988). Random monotone data fit simple algebraic models: Correlation is not confirmation. Psychological Bulletin, 104, 417-423.

PAVEL, M., \& Iverson, G. J. (1981). Invariant characteristics of partial masking: Implications for mathematical models. Journal of the Acoustical Society of America, 69, 1126-1131.

Penner, M. J., \& LARKIN, W. D. (1989). A model of sensation matching applied to recruitment. Manuscript in preparation.

PertovaAra, A. (1979). Modification of human pain threshold by specific tactile receptors. Acta Physiologica Scandinavica, 107, 339-341.

Pfanzagl, J. (1968). Theory of measurement. New York: Wiley.

PFeiffer, E. A. (1968). Electrical stimulation of sensory nerves with skin electrodes for research, diagnosis, communication, and behavioral conditioning: A survey. Medical \& Biological Engineering, 6, 637-651.

Phillips, D. P. (1987). Stimulus intensity and loudness recruitment: Neural correlates. Journal of the Acoustical Society of America, 82, $1-12$.

REgER, S. N. (1936). Differences in loudness response of the normal and hard-of-hearing ear at intensity levels slightly above threshold. Annals of Oto-Rhino-Laryngology, 45, 1029-1036.

Reilly, J. P., Freeman, V. T., \& Larkin, W. D. (1985). Sensory effects of transient electrical stimulation: Evaluation with a neuroelectric model. IEEE Transactions on Biomedical Engineering, BME32, 1001-1011.

ReILly, J. P., \& LARKIN, W. D. (1983). Electrocutaneous stimulation with high-voltage capacitive discharges. IEEE Transactions on Biomedical Engineering, BME-30, 631-641.

Robinson, D. W., \& DADSON, R. S. (1956). A re-determination of the equal-loudness relations for pure tones. British Journal of Applied Physics, 7, 166-181.

Rollman, G. B. (1974). Electrncutaneous stimulation. In F. A. Geldard (Ed.), Cutaneous communication systems and devices (pp. 3851). Austin, TX: Psychonomic Society.

Rollman, G. B. (1975). Behavioral assessment of peripheral nerve function. Neurology, 25, 339-342.

Salvi, R. J., Henderson, D., Hamerink, R., \& Ahroon, W. A. (1983). Neural correlates of sensorineural hearing loss. Ear \& Hearing, 4, 115-129. 
SANDERS, J. W. (1979). Recruitment. In W. F. Rintelman (Ed.), Hearing assessment (pp. 261-280). Baltimore: University Park Press.

SCHARF, B. (1976). Acoustic reflex, loudness summation, and the critical band. Journal of the Acoustical Society of America, 60, 753-755.

SCharf, B. (1978). Loudness. In E. C. Carterette \& M. P. Friedman (Eds.), Handbook of perception: Vol. 4. Hearing (pp. 187-242). New York: Academic Press.

SteInBERG, J. C., GARDNer, M. B. (1937). The dependence of hearing impairment on sound intensity. Joumal of the Acoustical Society of America, 9, 11-23.

STEVens, S. S. (1955). The measurement of loudness. Journal of the Acoustical Society of America, 27, 815-829.

SteVENS, S. S. (1966). Power-group transformations under glare, masking, and recruitment. Journal of the Acoustical Society of America, 39, 725-735.

Stevens, S. S. (1975). Psychophysics: Introduction to its perceptual, neural, and social prospects. New York: Wiley.

Stevens, S. S., \& Greenbaum, H. B. (1966). Regression effect in psychophysical judgment. Perception \& Psychophysics, 1, 439-446.

Stevens, S. S., Guirao, M. (1967). Loudness functions under inhibition. Perception \& Psychophysics, 2, 459-465.

SuPPES, P., \& Zinnes, J. L. (1963). Basic measurment theory. In R. D. Luce, R. R. Bush, \& E. H. Galanter (Eds.), Handbook of mathematical psychology (Vol. 1, pp. 1-76). New York: Wiley.

Teghtsoonian, R. (1971). On the exponents in Steven's law and the constant in Ekman's law. Psychological Review, 78, 71-80.

ThalmanN, R. (1965). Cross-modality matching in the study of abnormal loudness functions. Laryngoscope, 75, 1708-1726.

Treisman, M. (1964). Sensory scaling and the psychophysical law. Quarterly Joumal of Experimental Psychology, 16, 11-12.

Wall, P. D., \& Cronly-Dillon, J. R. (1960). Pain, itch, and vibration. Archives of Neurology, 2, 365-375.

\section{NOTES}

1. We refer to the "steepness" of Figures 2 and 3, and of analogous plots of loudness-balance functions, as an expedient way of describing the data. It is common practice, in psychophysics and audiology, to use the term "slope," either in its intuitive, visual sense, or in the sense of an exponent of a fitted power function. We avoid this usage throughout this paper. It should be recognized that a "straight line" in logarithmic coordinates (a power function) usually does not have a constant rate of change, and therefore cannot be characterized as having a "slope." We shall use the term "slope" only in its technical sense as the first derivative of a function. This terminological restraint is necessary be- cause, as can be seen in comparing Figures 2 and 3 with Figure 4, the visual apprehension of the relative rates of change of two variables can be misleading.

2. Our use of $r^{2}$ is not intended as an unconditional reliance on this statistic as an index of linearity, but as a useful descriptive measure that should be interpreted with caution. Birnbaum (1973) has discussed some of the reasons for caution. Parker, Casey, Ziriax, and Silberberg (1988) have shown that high values of $r^{2}$ are implied merely by an hypothesis of monotonicity. It is not clear, however, that any purely statistical approach to assessing the adequacy of a straight line can be sufficient in itself.

3. The correspondence between Teghtsoonian's (1971) finding and the range-dependent character of Equation 7 is only schematic, rather than quantitative, because we use a linear measure of the stimulus and of the stimulus range, whereas Teghtsoonian measured both logarithmically (see Note 1).

4. Two parameters, $\alpha$ and $\beta$, rather than just $\beta$ alone, are needed to represent the power transformation for response bias. The second parameter, $\alpha$, is required to ensure dimensional invariance. For Equation 8 to be meaningful, it must accommodate multiplicative changes in the units of $X_{1}$ and $X_{2}$ (e.g., transformations from sound pressure measured in pascals to sound pressure measured in dynes $/ \mathrm{cm}^{2}$ ) (see Luce, 1978; Pfanzagl, 1968; Suppes \& Zinnes, 1963). It is easy to verify that without $\alpha$, which has the units of $X^{1-\beta}$, Equation 8 would not meet the requirement for dimensional invariance.

As an alternative to Equation 8, one might apply the biasing transformation directly to the dimensionless quantities in Equation 6 . This alternative is discussed in Penner and Larkin (1989).

5. Thalmann (1965) displayed his data on graphs of decibels "hearing level," as is common practice in audiology. His individual subjects' thresholds differed from the audiometric standard by no more than $3 \mathrm{~dB}$. Thus, in plotting his group data on a decibel scale of "sensation level" in Figure 8, we risk an error corresponding to the (unknown) deviation of the group threshold from the audiometric standard. This error is likely to be small, and merely shifts the data uniformly in a diagonal direction on the plot.

The narrowband data for Hellman et al. (1987), plotted as solid points in Figure 8, show the condition in which their subjects adjusted the noisemasked stimulus. Data for the condition in which their subjects adjusted the tone in quiet were essentially identical. For the experiments with wideband noise, the two conditions gave slightly different results. The open circles in Figure 8 represent an average of these two conditions.

(Manuscript received July 13, 1987; revision accepted for publication February 13, 1989.) 